\title{
Kernel Function
}

National Cancer Institute

\section{Source}

National Cancer Institute. Kernel Function. NCI Thesaurus. Code C78544.

A function that measures the distance between two expression vectors as the data are projected into higher-dimensional space. 